\title{
Saddle Point Non-Singular Value Solution Based on Generalized Inverse Hermitian Triangulation and Split Iteration
}

\author{
LiJuan
}

Longnan Teachers College,Longnan 742500,China

Keywords: Saddle point system, Split iteration, Generalized anti Hermitian, Convergence, Non-singular.

\begin{abstract}
In order to achieve the nonsingular solution of saddle point linear system, this paper proposes an improved nonlinear Uzawa splitting iteration method, and combined with the generalized anti Hermitian triangle method, the algorithm is further modified, to improves the convergence of the algorithm. Finally, using the GMRES method and block diagonal Hermitian constraints carry out solve for the saddle point linear system, and then this paper can obtain the comparison results of saddle point coefficient matrix spectrum and calculation speed and accuracy. It can be seen from the calculation results that combined with the generalized inverse Hermitian triangulation method, the modified nonlinear Uzawa splitting iteration method has faster calculation speed and better convergence.
\end{abstract}

\section{Introduction}

In many complex scientific and engineering fields, the use of large sparse linear systems will generate a saddle point linear structure, which has great significance for the study of saddle point linear systems singular solutions [1]. For solving the saddle point linear system, many iterative algorithms and preconditioning algorithm are used in the saddle point solution method, they also made a certain effect, but these methods are often aimed at saddle point nonsingular solution [2]. How to deal with the non singular saddle point problem, there are better algorithm efficiency and accuracy, and combined with the stoke equations of computational fluid dynamics, this paper will do further research.

\section{Saddle Point Structure Computational Fluid Linear System}

In many engineering and science areas, there exists the saddle point structure of linear systems, in which mainly includes the constraint conditions optimization, control optimization, weighted least square optimization, numerical and fluid calculation optimization [3]. Saddle point linear system is generated by discrete Stokes equation or the second order elliptic equation, and generating process is generally produced in the use of the finite difference or finite element process [4]. In order to calculate Stoke equations as an example in the circulation, this study introduces the saddle point system. The hypothesis $\Omega$ is $R^{2}$ or $R^{3}$ to solve a range and the range is connected region. The use of the constraint condition on $\partial \Omega$ boundary, given field function is $f$, we can solve force field function $p$ and velocity field function $u$ by the formula (1).

$$
\begin{aligned}
\alpha u_{t}-v \Delta u+(u . \nabla) u+\nabla p & =f, \quad \text { in } \Omega \\
-\nabla \cdot u & =0, \quad \text { in } \Omega
\end{aligned} .
$$

Wherein $v$ represents the viscosity coefficient. When $\alpha=0$, the formula (1) can be written in the steady-state Stokes equation.

$$
\begin{aligned}
-v \Delta u+(u \cdot \nabla) u+\nabla p=f, & \text { in } \Omega \\
-\nabla \cdot u=0, & \text { in } \Omega
\end{aligned} .
$$


If only considering the formula (2), assuming an arbitrary initial vector $u^{(0)}$ in the case of $m=0,1,2, \ldots$, carries out the iterative calculation for the formula (2). The iterative method selects the Picard form, we can get

$$
\begin{array}{r}
-v \Delta u^{(m+1)}+\left(u^{(m)} \cdot \nabla\right) u^{(m+1)}+\nabla p^{(m+1)}=f, \quad \text { in } \Omega \\
-\nabla \cdot u^{(m+1)}=0, \quad \text { in } \Omega
\end{array} .
$$

In formula (3), the vector $w$ is known field, and the field is Picard iteration and divergence free velocity field. If meeting $w=0$ conditions, using finite element or finite difference carry out discrete for the formula (3), then we can get the saddle point linear system.

$$
A u \equiv\left(\begin{array}{cc}
M & E \\
-E^{*} & 0
\end{array}\right)\left(\begin{array}{l}
u_{1} \\
u_{2}
\end{array}\right)=\left(\begin{array}{l}
f_{1} \\
f_{2}
\end{array}\right) \equiv f .
$$

Wherein $M \in C^{p \times p}$ represents the Hermitian positive definite matrix; $E \in C^{p \times p}$ represents that matrix satisfies $q \leq p ; f \in C^{p+q}$ represents matrix coefficient and shows the known vector in the computational fluid space numerical region, it satisfies the condition $A \in C^{(p+q) \times(p+q)}$, and there are $A \in C^{p}$ and $f_{2} \in C^{q}$.

\section{The Saddle Point Singular Value Solution Based on the Generalized Inverse Hermitian Triangulation and Split Iteration}

This research mainly adopts split iteration method and generalized anti Hermitian triangle method, to calculate the singular solutions of saddle point [5]. In order to study the generalized saddle point problem, we establish the mathematical model of saddle point problems. The formula is as follows:

$$
A u=\left(\begin{array}{cc}
A & B^{T} \\
B & -C
\end{array}\right)\left(\begin{array}{l}
x \\
y
\end{array}\right)=\left(\begin{array}{l}
f \\
g
\end{array}\right)=b .
$$

Wherein $A \in R^{n \times n}$ has positive definite, and $C \in R^{m \times m}$ has positive semi definite. For $B \in R^{m \times n}, m \leq n$, in which $B^{T}$ is the transpose matrix of $B$. For $f \in R^{n}, g \in R^{m}$, the system is very important for a class of numerical calculation linear system, we can use the Uzawa algorithm of iterative splitting to solve the problem. The assumption of any given $x_{0} \in R^{n}$ and $y_{0} \in R^{m}$, the sequence of iterations $\left\{\left(x_{i}, y_{i}\right)\right\}(i=0,1, \ldots$,$) is defined as$

$$
\left\{\begin{array}{c}
x_{i+1}=x_{i}+A^{-1}\left(f-A x_{i}-B^{T} y_{i}\right) \\
y_{i+1}=y_{i}+T Q_{B}^{-1}\left(B x_{i+1}-C y_{i}-g\right)
\end{array} .\right.
$$

Wherein $\tau$ represents real parameters, $Q_{B}$ represents positive definite matrix, and its satisfaction is

$$
\gamma\left(Q_{B} w, w\right) \leq\left(\left(B(A)^{-1} B^{T}+C\right) w, w\right) \leq\left(Q_{B} w, w\right), \forall w \in R^{m} .
$$

The error of the iterative calculation is

$$
e_{i}^{x}=x-x_{i}, e_{i}^{v}=y-y_{i} \text {. }
$$

For the non symmetric saddle point problems, combined with the generalized anti Hermitian triangle method, we consider the different constraint conditions, such as block diagonal or triangular block, we can expand the calculation conditions and constraint conditions. Finally, through the pre-processing matrix spectrum, this paper is to characterize the saddle point of the singular solution.

To consider the following diagonal block constraints, there is

$$
\varsigma=\left(\begin{array}{cc}
G & 0 \\
0 & C G^{-1} B^{T}
\end{array}\right) \text {. }
$$


Wherein $G-E=A$ represents a non singular split matrix, which is a block diagonal pretreatment matrix eigenvalue analysis. Its constraints can also be written

$$
P=\left(\begin{array}{cc}
G & 0 \\
0 & -C G^{-1} B^{T}
\end{array}\right) .
$$

In the case of $G=A$, we can get the exact block constraints. This paper presents a very similar constraint with the previous introduction.

$$
P_{a . b}=\left(\begin{array}{cc}
G & a B^{T} \\
0 & b C G^{-1} B^{T}
\end{array}\right) .
$$

The constraint is based on generalized anti Hermitian triangle method, it not only can precise processing matrix characteristic value, but also can handle the perturbations of non exact constraint conditions, and its calculation results is better than above method.

\section{Saddle Point Generalized Anti Hermitian Triangle Method and Iterative Splitting Solution}

For solving the saddle point system, it can be combined with generalized inverse Hermitian triangulation method and iterative split Uzawa algorithm. Considering different constraints, it can go through the pretreatment matrix spectrum to characterize the singular solutions of saddle point [6-8]. Combined with the constraint conditions GMRES method, the examples can solve the saddle point system, in which the saddle point system is

$$
A x=b \text {. }
$$

Among them, assuming that the values of $b$ can make the values of $x$ is 1 , we can use zero vectors as the initial vector, the iteration termination condition is set to calculate the number of more than 50 , and the convergence precision is set to

$$
\frac{\|b-A x k\|_{2}}{\|b\|_{2}} \leq 10^{-6} \text {. }
$$

Considering the example has appropriate boundary conditions, we can convert it to Oseen problem.

$$
\left\{\begin{array}{c}
-v \Delta u+w \cdot \nabla u+\nabla p=f, i n \Omega \\
\operatorname{div} u=0, i n \Omega
\end{array} .\right.
$$

Wherein the vector function $u$ is expressed in the $\Omega$ upper speed, and $w$ meets $\nabla \cdot w=0$. Using square element $n \times n$ grid and discrete solution method, we can obtain matrix $\hat{A}$.

$$
\widehat{A}=\left(\begin{array}{ccc}
F 1 & & B_{u}^{T} \\
& F 2 & B_{v}^{T} \\
B u & B v & 0
\end{array}\right) .
$$

Among them, $\left(\begin{array}{ll}F_{1} & \\ & F_{2}\end{array}\right)=A$ has positive definite. This calculation uses the $18 \times 18$ grid, we can structure matrix $\hat{A}$ through the $A$. The saddle point coefficient matrix is obtained by the calculation as shown in Figure 1. 


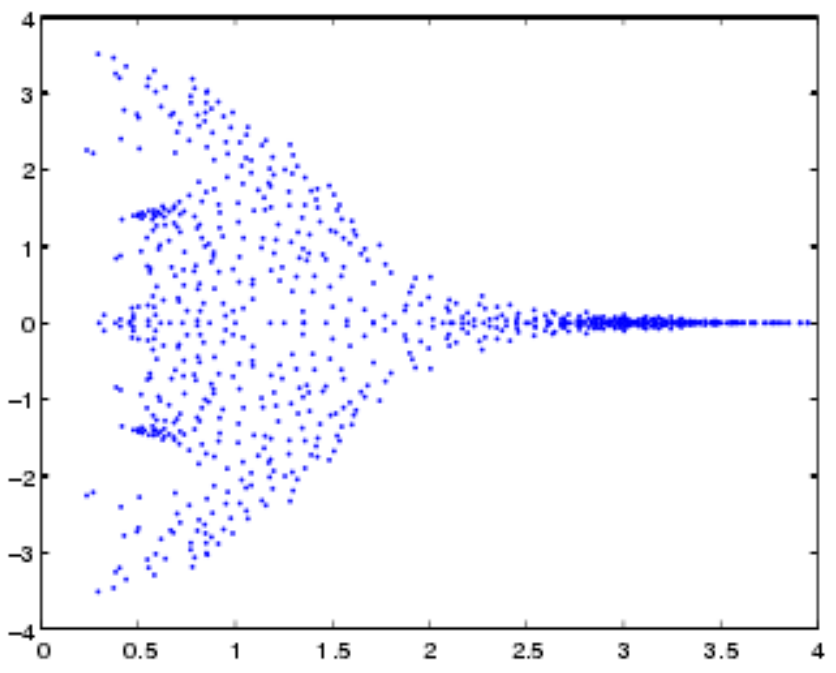

(a) $\operatorname{Nullity}(A)=0$

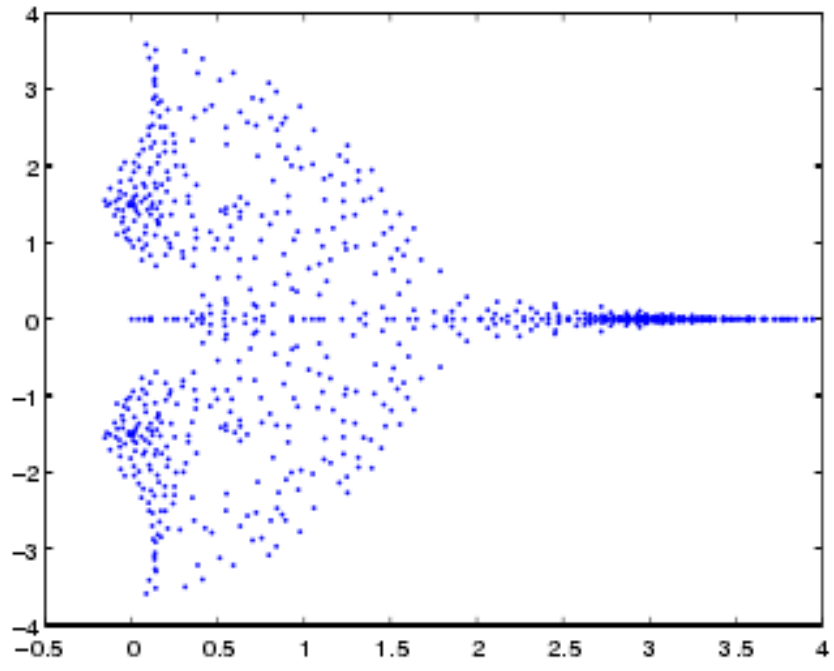

(b) Nullity (B) $=0.5 \mathrm{~m}$

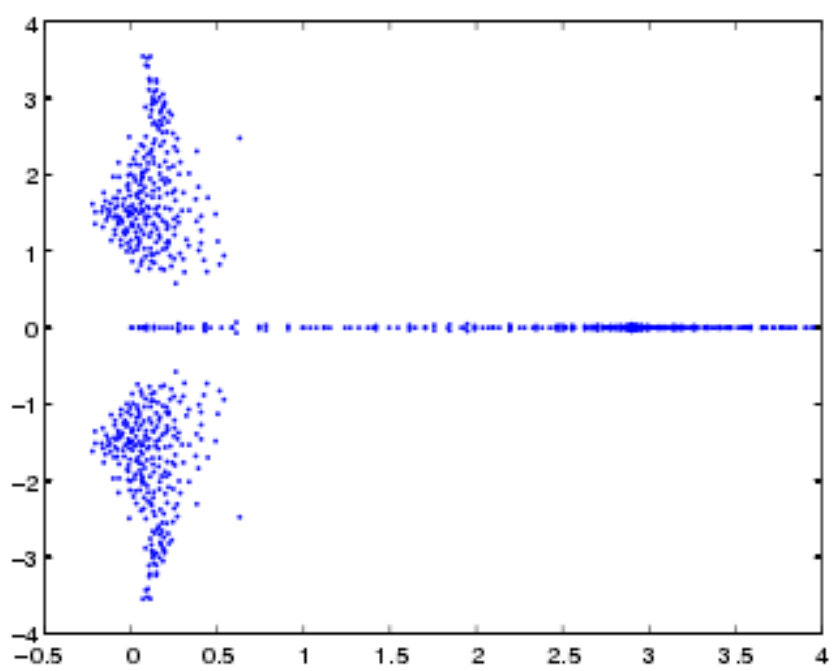

(c) Nullity (C) $=5 \mathrm{~m}$

Fig. 1 Saddle point coefficient matrix spectra

Figure 1 shows the matrix spectra of the saddle point coefficients obtained by calculating. In Figure 1, we can calculate the spectral distribution of the original matrix spectrum $A i$ for a matrix $A$ with 
different zero degrees. The result shows that the generalized anti Hermitian triangle has higher zero degree, and the characteristic value of the coefficient matrix is more concentrated.

Table 1. Comparison of calculation accuracy

\begin{tabular}{|c|c|c|}
\hline Algorithm & The number of iterations & Calculation precision \\
\hline $\begin{array}{c}\text { Generalized inverse Hermitian triangle and } \\
\text { Uzawa splitting iteration }\end{array}$ & 65 & 0.001 \\
\hline Block diagonal preconditioner constraint & 125 & 0.01 \\
\hline
\end{tabular}

Through the calculated precision comparison table 1, it can be seen that using generalized anti Hermitian triangular with Uzawa splitting iterative algorithm, iterative steps are only 65, it is lose to calculate the half the time introduced by the second section algorithm, and its calculation accuracy is 0.001, which is more than ten times introduced by the second section algorithm, there is fast calculation speed and high precision.

\section{Summary}

In order to solve the solution of the saddle point linear system in the non singular case, this paper proposes a modified Uzawa splitting iterative algorithm, and combined with Hermitian block diagonal constraints, this paper calculates the coefficient matrix spectrum of saddle point system and the convergence. The calculation results show that combined Uzawa splitting iterative algorithm, using the generalized anti Hermitian triangular for computing time is only half of the block diagonal constraint, and its calculation accuracy is 10 times of the block diagonal preconditioning, those show that this paper proposes algorithm with fast calculation speed and good convergence characteristics, and there are good numerical computational advantages.

\section{References}

[1] M. Zhang, S.R. Sun, Y.G.. Zhao. Positive solutions of boundary value problems for a class of fractional differential equation existence. Jinan University Journal (NATURAL SCIENCE EDITION), 2014, 24(2): 205-210.

[2] S.C. Li. The similar structure of solutions to the boundary value problem for second-order linear homogeneous differential equation. Journal of Xihua University (NATURAL SCIENCE EDITION), 2014, 26(1): 84-86.

[3] J.L. Li, T.Z. Huang, L. Li. The spectral properties of the preconditioned matrix for nonsymmetrical saddle point problems. Journal of computational and applied mathematics, 2014, 23(5): 270-285.

[4] G..F. Zhang, Q.H. Lu. On generalized symmetric SOR method for augmented systems. J. Com-put. Appl. Math., 2013, 21(9): 51-58.

[5] B. Zheng, Z.Z. Bai, X. Yang. On semi-convergence of parameterized Uzawa methods for singular saddle point problems. Linear Algebra Appl., 2013, 4(31): 808-817.

[6] M. ur Rehman, C. Vuik, G. Segal. SIMPLE-type preconditioners for the Oseen problem. Int. J.Numer. Meth. Fluids., 2013(61): 432-452.

[7] X.H. Shao, Z. Li, C.J. Li. Modified SOR-like method for the augmented system. Intern J. Computer. Math., 2013(84): 1653-1662.

[8] T. Rees, C. Greif. A preconditioner for linear systems arising from interior point optimizationmethods. SIAM J. Sci. Comput., 2013(29): 1992-2007. 